DESY Report 16-231

\title{
Dipole model analysis of highest precision HERA data, including very low $Q^{2}$ 's
}

\author{
A. Luszczak $^{1}$, H. Kowalski ${ }^{2}$ \\ 1 T.Kosciuszko Cracow University of Technology, 30-067 Cracow, Poland \\ 2 Deutsches Elektronen-Synchrotron DESY, D-22607 Hamburg, Germany
}

\begin{abstract}
We analyse, within a dipole model, the final, inclusive HERA DIS cross section data in the low $x$ region, using fully correlated errors. We show, that these highest precision data are very well described within the dipole model framework starting from $Q^{2}$ values of $3.5 \mathrm{GeV}^{2}$ to the highest values of $Q^{2}=250 \mathrm{GeV}^{2}$.

To analyze the saturation effects we evaluated the data including also the very low $0.35<Q^{2} \mathrm{GeV}^{2}$ region. The fits including this region show a preference of the saturation ansatz.
\end{abstract}

\section{Introduction}

In our last paper [1] we investigated the high precision, inclusive HERA data [2] within the dipole model. In the present paper we continue this investigation using the highest precision data [3], evaluated with the correlated errors.

Many investigations have shown that HERA inclusive and diffractive DIS cross sections are very well described by the dipole models [4, 6], which provide a natural description of QCD reaction in the low- $x$ and low $Q^{2}$ region. They allow a simultaneous description of many different physics reactions, like inclusive DIS processes, inclusive diffractive processes, exclusive $J / \psi, \rho, \phi$ production, diffractive jet production, or diffractive and non-diffractive charm production. Due to the optical theorem, all these processes are determined by the same, universal, gluon density [7 9]. The understanding of the properties of the gluon density and its precise knowledge is very important because the QCD-evolved gluon density determines the cross sections of most relevant physics processes, e.g. Higgs production at LHC. Any significant deviation of the predicted cross section from their Standard Model value could be a sign of new physics. 
The precise determination of the gluon density relies on the analysis of high quality, inclusive, DIS data taken over the full, experimentally accessible $x$ and $Q^{2}$ region. Therefore, the H1 and ZEUS experiments have combined their inclusive DIS cross sections which, due to a substantial reduction of systematic measurements errors, led to an increase of precision by about a factor two [2]. More recently, also the final set of HERA data was released which provides a full set of correlated errors. These errors contain the most complete information about the data and lead to very restrictive fits [3]. The full information about the data is made now accessible within the xFitter facility [10].

The aim of this paper is to investigate the additional information contained in the final HERA data. The most precise data where obtained in the region of higher $\mathrm{Q}^{2}$ 's $\left(\mathrm{Q}^{2}\right.$ from 3.5 to $\mathrm{O}(10000) \mathrm{GeV}^{2}$ ), where the DGLAP evolution is known to describe data very well. In the low $x$ region, which is investigated here, the highest achievable $Q^{2}$ is around $250 \mathrm{GeV}^{2}$. The investigation is performed using the so called BGK model, see below, which uses the DGLAP evolution in the dipole scheme. The dipole approach allows to extend the perturbative description to the region of much smaller $Q^{2}$ 's, $0.35<Q^{2}<3.5 \mathrm{GeV}^{2}$. The simultaneous evaluation of the very precise data at higher $Q^{2}$ together with the low $Q^{2}$ data allows to address again the question of high density gluonic states.

The paper is organized as follows: in Section 2 we recall the main properties of the dipole approach and review the dipole models. In Section 3 we present the results of the dipole and pdf fits in the higher $3.5<Q^{2}<250 \mathrm{GeV}^{2}$ region. In Section 4 we discuss saturation effects including data in the lower $Q^{2}$ region. In Section 5 we summarize the results.

\section{Dipole models}

The dipole picture was first derived, in the low $x$ limit of QCD, by Nikolaev and Zaharov [13. They have shown that the deep inelastic scattering can be viewed as a two stage process; first the virtual photon fluctuates into a dipole, which consists of a quark-antiquark pair (or a $q \bar{q} g$ or $q \bar{q} g g$... system) and in the second stage the dipole interacts with the proton. Dipole denotes a quasi-stable quantum mechanical state, which has a very long life time $\left(\approx 1 / m_{p} x\right)$ and a size $r$, which remains unchanged during scattering. The wave function $\Psi$ determines the probability to find a dipole of size $r$ within a photon. This probability depends on the value of external $Q^{2}$ and the fraction of the photon momentum carried by the quarks forming the dipole, $z$. Neglecting the $z$ dependence, in a very rough approximathion, $Q^{2} \sim 1 / r^{2}$.

The scattering amplitude is a product of the virtual photon wave function, $\Psi$, with the dipole cross section, $\sigma_{\text {dip }}$, which determines a probability of the dipole-proton scattering. Thus, within the dipole formulation of the $\gamma^{*} p$ scattering

$$
\sigma_{T, L}^{\gamma^{*} p}\left(x, Q^{2}\right)=\int d r^{2} \int d z \Psi_{T, L}^{*}(Q, r, z) \sigma_{\operatorname{dip}}(x, r) \Psi_{T, L}(Q, r, z)
$$

where $T, L$ denotes the virtual photon polarization and $\sigma_{T, L}^{\gamma^{*} p}$ the total inclusive DIS cross section. 
This simple and intuitive approach became then a basis of many dipole many models, [14 20]. which have been developed to test various aspects of data. They vary due to different assumption made about the physical behavior of dipole cross sections. In the following we will shortly review some them to motivate the choice of the model used for present investigation.

\section{$2.1 \quad$ GBW model}

The dipole model became an important tool in investigations of deep-inelastic scattering due to the initial observation of Golec-Biernat and Wüesthoff (GBW) [4], that a simple ansatz for the dipole cross section was able to describe simultaneously the total inclusive and diffractive cross sections.

In the GBW model the dipole-proton cross section $\sigma_{\text {dip }}$ is given by

$$
\sigma_{\text {dip }}\left(x, r^{2}\right)=\sigma_{0}\left(1-\exp \left[-\frac{r^{2}}{4 R_{0}^{2}(x)}\right]\right),
$$

where $r$ corresponds to the transverse separation between the quark and the antiquark, and $R_{0}^{2}$ is an $x$ dependent scale parameter which has a meaning of saturation radius, $R_{0}^{2}(x)=$ $\left(x / x_{0}\right)^{\lambda_{G B W}} / \mathrm{GeV}^{-2}$. The free fitted parameters are: the cross-section normalisation, $\sigma_{0}$, as well as $x_{0}$ and $\lambda_{G B W}$. In this model saturation is taken into account in the eikonal approximation and the saturation radius is intimately related to the gluon density, see below. The exponent $\lambda_{G B W}$ determines the growth of the total and diffractive cross section with decreasing $x$. For dipole sizes which are large in comparison to the saturation radius, $R_{0}$, the dipole cross section saturates by approaching a constant value $\sigma_{0}$, i.e. saturation damps the growth of the gluon density at low $x$.

The GBW model provided a good description of data from medium $Q^{2}$ values $(\approx 30$ $\left.\mathrm{GeV}^{2}\right)$ down to low $\left.Q^{2}(\approx 0.1) \mathrm{GeV}^{2}\right)$. Despite its success and its appealing simplicity the model has some shortcomings; in particular it describes the QCD evolution by a simple $x$ dependence, $\sim(1 / x)_{B G W}^{\lambda}$, i.e the $Q^{2}$ dependence of the cross section evolution is solely induced by the saturation effects. Therefore, it does not match with DGLAP QCD evolution, which is known to describe data very well from $Q^{2} \approx 4 \mathrm{GeV}^{2}$ to very large $Q^{2} \approx 10000 \mathrm{GeV}^{2}$.

\subsection{BGK model}

The evolution ansatz of the GBW model was improved in the model proposed by Bartels, Golec-Biernat and Kowalski, (BGK) [5], by taking into account the DGLAP evolution of the gluon density in an explicit way. The model preserves the GBW eikonal approximation to saturation and thus the dipole cross section is given by

$$
\sigma_{\text {dip }}\left(x, r^{2}\right)=\sigma_{0}\left(1-\exp \left[-\frac{\pi^{2} r^{2} \alpha_{s}\left(\mu^{2}\right) x g\left(x, \mu^{2}\right)}{3 \sigma_{0}}\right]\right) .
$$

The evolution scale $\mu^{2}$ is connected to the size of the dipole by $\mu^{2}=C / r^{2}+\mu_{0}^{2}$. This assumption allows to treat consistently the contributions of large dipoles without making 
the strong coupling constant, $\alpha_{s}\left(\mu^{2}\right)$, un-physically large. This means also that we can extend the model, keeping its perturbative character, to the data at low $Q^{2}$, because the external $Q^{2}$ and the internal $\mu^{2}$ scales are connected only by the wave function.

The gluon density, which is parametrized at the starting scale $\mu_{0}^{2}$, is evolved to larger scales, $\mu^{2}$, using LO or NLO DGLAP evolution. We consider here two forms of the gluon density:

- the soft ansatz, as used in the original BGK model

$$
x g\left(x, \mu_{0}^{2}\right)=A_{g} x^{-\lambda_{g}}(1-x)^{C_{g}},
$$

- the soft + hard ansatz

$$
x g\left(x, \mu_{0}^{2}\right)=A_{g} x^{-\lambda_{g}}(1-x)^{C_{g}}\left(1+D_{g} x+E_{g} x^{2}\right),
$$

The free parameters for this model are $\sigma_{0}$ and the parameters for gluon $A_{g}, \lambda_{g}, C_{g}$ or additionally $D_{g}, E_{g}$, Their values are obtained by a fit to the data. The fit results were found to be independent on the parameter $C$, which was therefore fixed as $C=4 \mathrm{GeV}^{2}$, in agreement with the original BGK fits. It is also possible to vary the parameter $\mu_{0}^{2}$. However, to assure that the evolution is performed in the perturbative region and to be compatible with the standard pdf fits we took as a starting scale $\mu_{0}^{2}=1.9$ or $1.1 \mathrm{GeV}^{2}$. In the BGK model, the $\mu_{0}^{2}$ scale is the same as the $Q_{0}^{2}$ scale of the standard QCD pdf fits.

\section{Results of fits in the higher $Q^{2}$ region}

This paper concentrates on the inclusive DIS measurements in the low $x$ region, $x<0.01$. Here, the contribution of the valence quarks is small, below $7 \%$, and has therefore been neglected for a long time. However now, the combined H1 and ZEUS HERA data achieve a precision of about $2 \%$. Theoretically, it is very difficult to treat valence quarks inside the dipole framework because, the dipole amplitudes are not well defined in the region of high $x$. In our previous paper [1], we developed therefore an heuristic approach in which we added the valence quark contribution from the standard pdf's fits to the dipole predictions. Hence, the dipole contribution plays just a role of the sea quarks in the standard pdf's. This procedure is justified by the fact that the sea quark contribution disappears at larger $x$.

For the purpose of this investigation we choose the BGK model, because it is expected to provide the best description of data in the higher $Q^{2}$ range, as it uses DGLAP evolution. The fits were performed within the xFitter system, where the dipole model and the valence quarks contributions are a part of the same framework [10]. Therefore, the QCD evolution is the same as in the standard xFitter pdf fits. For gluon density we used both the soft and soft+hard ansatz with the NLO evolution.

We used for fits a complete set of reduced cross section points, $\sigma_{r}$, obtained at different electron and proton energies: HERA1+2-NCep-460, HERA1+2-NCep-575, HERA1+2NCep-820, HERA1+2-NCep-920 and HERA1+2-NCem. 


\subsection{Fits to the dipole model.}

The results of the BGK fit with valence quarks and the soft gluon density, are shown in Table 1, 2 and 3. The best fit is obtained using valence quarks and gluon density of the soft + hard type, see Table 4 and 5. In Table 1, 2 and 4 the starting QCD scale is $Q_{0}^{2}=1.9$ $\mathrm{GeV}^{2}$, in Table 5 it is $Q_{0}^{2}=1.1 \mathrm{GeV}^{2}$,

In Table 1, 4 and 5 the valence quark contribution was taken from the standard pdf fit, shown below, in Table 2 the valence quarks were fitted. In these tables, $N_{d f}$ denotes the number of degrees of freedom, which is equal to the number of measured data points minus the number of free parameters used in the fit. The parameters $\sigma_{0}$ of the dipole model and the starting parameters for gluon $A_{g}, \lambda_{g}, C_{g}$ are obtained from the fit. The value of the parameter $C$ was fixed, as explained above. To limit the fit to the perturbative region only we took $Q^{2} \geq Q_{\min }^{2}$ with $Q_{\min }^{2}=3.5$ (or 8.5) $\mathrm{GeV}^{2}$. For the $x$ region, we took $x \leq 0.01$. There are 538 (or 452) measured points in this region.

\begin{tabular}{|c|c|c|c|c|c|c|c|}
\hline$Q_{\min }^{2}\left[\mathrm{GeV}^{2}\right]$ & $\sigma_{0}[\mathrm{mb}]$ & $A_{g}$ & $\lambda_{g}$ & $C_{g}$ & $N_{d f}$ & $\chi^{2}$ & $\chi^{2} / N_{d f}$ \\
\hline 3.5 & $87.0 \pm$ & $2.32 \pm$ & $-0.056 \pm$ & $8.21 \pm$ & 534 & 551.1 & 1.03 \\
& 8.9 & 0.009 & 0.11 & 0.80 & & & \\
8.5 & $72.4 \pm$ & $2.77 \pm$ & $-0.042 \pm$ & $6.54 \pm$ & 448 & 452.5 & 1.01 \\
& 7.4 & 0.009 & 0.123 & 0.632 & & & \\
\hline
\end{tabular}

Table 1: BGK fit with fixed valence quarks for $\sigma_{r}$ for H1ZEUS-NC data in the range $Q^{2} \geq 3.5$ or $8.5 \mathrm{GeV}^{2}$ and $x \leq 0.01$. NLO fit. Soft gluon. $m_{u d s}=0.14, m_{c}=1.3 \mathrm{GeV}$. $Q_{0}^{2}=1.9 \mathrm{GeV}^{2}$.

\begin{tabular}{|c|c|c|c|c|c|c|c|}
\hline$Q_{\min }^{2}\left[\mathrm{GeV}^{2}\right]$ & $\sigma_{0}[m b]$ & $A_{g}$ & $\lambda_{g}$ & $C_{g}$ & $N_{d f}$ & $\chi^{2}$ & $\chi^{2} / N_{d f}$ \\
\hline 3.5 & $89.99 \pm$ & $2.44 \pm$ & $-0.079 \pm$ & $7.24 \pm$ & 530 & 540.35 & 1.02 \\
& 9.2 & 0.145 & 0.099 & 0.61 & & & \\
\hline
\end{tabular}

Table 2: BGK fit with fitted valence quarks for $\sigma_{r}$ for H1ZEUS-NC data in the range $Q^{2} \geq 3.5 \mathrm{GeV}^{2}$ and $x \leq 0.01$. NLO fit. Soft gluon. $m_{u d s}=0.14, m_{c}=1.3 \mathrm{GeV} . Q_{0}^{2}=1.9$ $\mathrm{GeV}^{2}$.

\begin{tabular}{|c||c||c||c|c||c|c|c||}
\hline No & Auv $($ fix $)$ & Buv & Cuv & Euv & $A d v($ fix $)$ & $B d v$ & $C d v$ \\
\hline 1 & 4.073 (sum rule) & $0.892 \pm$ & $5.832 \pm$ & $17.997 \pm$ & 3.151 (sum rule $)$ & $0.840 \pm$ & $3.480 \pm$ \\
& & 0.019 & 0.341 & 0.876 & & 0.012 & 0.056 \\
\hline
\end{tabular}

Table 3: Parameters of the valence quark contribution fitted in the BGK fit of Table 2 ,

In our previous work [1, which used a subset of data from the present evaluation and had a much poorer evaluation of errors, the differences between the fits with and without valence quarks were quite pronounced. Therefore, in Table 6 we show a BGK fit without the valence quarks. We observe that the fit is only slightly worse than the one with the fixed 


\begin{tabular}{|c|c|c|c|c|c|c|c|c|c|}
\hline$Q_{\min }^{2}\left[\mathrm{GeV}^{2}\right]$ & $\sigma_{0}[\mathrm{mb}]$ & $A_{g}$ & $\lambda_{g}$ & $C_{g}$ & $D_{g}$ & $E_{g}$ & $N_{d f}$ & $\chi^{2}$ & $\chi^{2} / N_{d f}$ \\
\hline 3.5 & $77,6 \pm$ & $2.62 \pm$ & $-0.064 \pm$ & $37.1 \pm$ & $3.06 \pm$ & $1406.4 \pm$ & 532 & 534.2 & 1.00 \\
& 18,6 & 0.16 & 0.0087 & 5.06 & 6.51 & 552.7 & & & \\
\hline 8.5 & $63.5 \pm$ & $2.11 \pm$ & $-0.054 \pm$ & $21.3 \pm$ & $1.10 \pm$ & $867.2 \pm$ & 448 & 439.0 & 0.98 \\
& 18.5 & 0.10 & 0.0065 & 4.062 & 5.76 & 423.7 & & & \\
\hline
\end{tabular}

Table 4: BGK fit with valence quarks for $\sigma_{r}$ for H1ZEUS-NC data in the range $Q^{2} \geq 3.5$ or $8.5 \mathrm{GeV}^{2}$ and $x \leq 0.01$. NLO fit. Soft + hard gluon. $m_{u d s}=0.14, m_{c}=1.3 \mathrm{GeV} . Q_{0}^{2}=1.9$ $\mathrm{GeV}^{2}$.

\begin{tabular}{|c|c|c|c|c|c|c|c|c|c|c|}
\hline$Q_{\min }^{2}\left[\mathrm{GeV}^{2}\right]$ & $Q_{0}^{2}\left[\mathrm{GeV}^{2}\right]$ & $\sigma_{0}[m b]$ & $A_{g}$ & $\lambda_{g}$ & $C_{g}$ & $D_{g}$ & $E_{g}$ & $N_{d f}$ & $\chi^{2}$ & $\chi^{2} / N_{d f}$ \\
\hline 3.5 & 1.1 & $220 \pm$ & $3.57 \pm$ & $0.082 \pm$ & $31.3 \pm$ & $11.0 \pm$ & $1360 \pm$ & 532 & 532 & 1.00 \\
& & 122 & 0.38 & 0.017 & 5.4 & 9.8 & 690 & & & \\
\hline
\end{tabular}

Table 5: BGK fit with valence quarks for $\sigma_{r}$ for H1ZEUS-NC data in the range $Q^{2} \geq$ $3.5 \mathrm{GeV}^{2}$ and $x \leq 0.01$. NLO fit. Soft + hard gluon. $m_{u d s}=0.14, m_{c}=1.3 \mathrm{GeV} . Q_{0}^{2}=1.1$ $\mathrm{GeV}^{2}$.

valence quarks, see Table1 and 2. The correlated errors of the final data are more restrictive than the uncorrelated one, as seen from the standard HERAPDF fits, however, in the case of dipole fits, the full treatment of errors makes the contribution of valence quarks less visible in the low $x$ region.

In Fig. 1 we show a comparison of the dipole BGK fit with valence quarks and soft+hard gluon density, Table 4 , with the data of HERA at $Q^{2}>3.5 \mathrm{GeV}^{2}$. For clarity only reduced cross section data taken with $E_{p}=920 \mathrm{GeV}$ is shown. Figure shows an excellent agreement with data.

\begin{tabular}{|c|c|c|c|c|c|c|c|}
\hline$Q_{\min }^{2}\left[\mathrm{GeV}^{2}\right]$ & $\sigma_{0}[\mathrm{mb}]$ & $A_{g}$ & $\lambda_{g}$ & $C_{g}$ & $N_{d f}$ & $\chi^{2}$ & $\chi^{2} / N_{d f}$ \\
\hline 3.5 & $105.20 \pm$ & $2.4788 \pm$ & $-0.066 \pm 3$ & $6.9093 \pm$ & 534 & 554.68 & 1.04 \\
& 12.234 & 0.093 & 0.004 & 0.510 & & & \\
\hline
\end{tabular}

Table 6: BGK fit without valence quarks for $\sigma_{r}$ for H1ZEUS-NC data in the range $Q^{2} \geq$ $3.5 \mathrm{GeV}^{2}$ and $x \leq 0.01$. NLO fit. Soft gluon. $m_{u d s}=0.14, m_{c}=1.3 \mathrm{GeV} . Q_{0}^{2}=1.9 \mathrm{GeV}^{2}$.

In this investigation the values of the light quark mass in the dipole formula were fixed to $0.14 \mathrm{GeV}$, like in the original GBW and BGK models. We performed also fits lowering the light quark masses but the resulting fits were of similar quality as the fits shown here.

\subsection{Results from the pdf fits}

In the Table 7 we show results of the standard HERAPDF fits [3] to the final HERA inclusive cross section data, which have fully correlated errors. They are performed in the same $\mathrm{Q}^{2}$ 


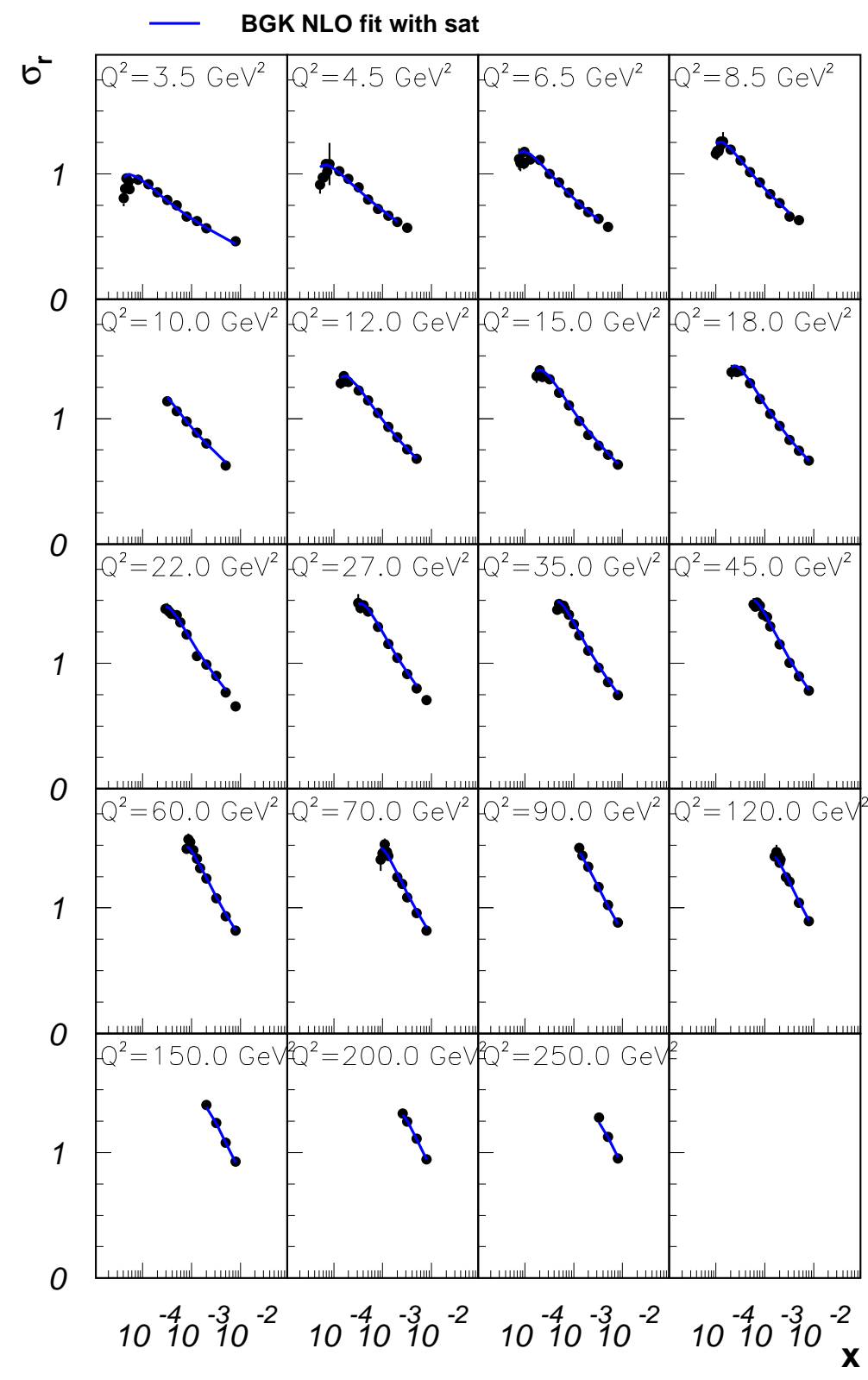

Figure 1: Comparison of the dipole BGK fit of Table 4 with the reduced cross sections of the final, combined H1 and ZEUS HERA data. For better visibility only $920 \mathrm{GeV}$ data are displayed. The fit was performed in the $Q^{2}>3.5 \mathrm{GeV}^{2}$ region. 
ranges as the dipole fits but in the full $x$ range. The full $x$ range is here necessary to fix the contribution of valence quarks. In Table 8 we show the parameters of the valence quarks obtained for $Q^{2}>3.5 \mathrm{GeV}^{2}$. These are the parameters used for the dipole fit with fixed valence quarks shown in Table 1 and 4 .

\begin{tabular}{|c|c|c|c|c|c|}
\hline No & $Q_{\min }^{2}\left[\mathrm{GeV}^{2}\right]$ & HF Scheme & $N p$ & $\chi^{2}$ & $\chi^{2} / N p$ \\
\hline 1 & 3.5 & RT & 1131 & 1356.70 & 1.20 \\
\hline 2 & 8.5 & RT & 456 & 470.88 & 1.15 \\
\hline
\end{tabular}

Table 7: HERAPDF NLO fits to the same data set as for the dipole model but in the full $x$ range. $Q_{0}^{2}=1.9 \mathrm{GeV}^{2}$.

\begin{tabular}{|c||c||c||c|c||c|c|c||}
\hline No & Auv & Buv & Cuv & Euv & $A d v$ & $B d v$ & $C d v$ \\
\hline 1 & $4.073 \pm$ & $0.713 \pm$ & $4.841 \pm$ & $13.405 \pm$ & $3.151 \pm 1$ & $0.806 \pm$ & $4.079 \pm$ \\
& 0.123 & 0.016 & 0.214 & 0.921 & 0.121 & 0.056 & 0.301 \\
\hline
\end{tabular}

Table 8: Parameters of valence quarks obtained in HERAPDF NLO fits for $Q^{2}>3.5 \mathrm{GeV}^{2}$.

\begin{tabular}{|c|c|c|c|c|c|}
\hline No & $Q_{\min }^{2}\left[\mathrm{GeV}^{2}\right]$ & HF Scheme & $N p$ & $\chi^{2}$ & $\chi^{2} / N p$ \\
\hline 1 & 3.5 & FONLL-B & 534 & 539,3 & 1.01 \\
2 & 3.5 & FONLL-B & 532 & 537,3 & 1.01 \\
\hline
\end{tabular}

Table 9: HERAPDF NLO fits with fixed valence quarks to the same data set as for the dipole model, but with $x<0.01$ range. $Q_{0}^{2}=1.9 \mathrm{GeV}^{2}$. No 1 soft gluon, No 2 soft + hard gluon

Table 7 shows that the standard HERAPDF fit is not describing data very well. The agreement improves somewhat when the fit is performed in a higher $Q^{2}$ range but it is still not fully satisfactory, as was extensively discussed in ref. [3]. We note that in case of the BGK dipole model the agreement with data is very good, see Table 1 and 2 . It is even slightly improving when a 5 parameter ansatz for gluon density, soft + hard, is used, see Table 4 and 5. The quality of a fit is not depending on the starting scale, an example of a fit with $Q_{0}^{2}=1.1 \mathrm{GeV}^{2}$ is shown in Table 5. In difference to the results of our previous paper [1], the dipole fit quality is not significantly improving with increasing $Q_{\text {min }}^{2}$, see Tables 1 and 4 .

It is also interesting to observe that in the low $x$ region, the dipole and HERAPDF fit have similar quality, see Table 4 and 9. The HERAPDF fits of Table 9 were performed with the fixed quark contribution and with the same ansatz for gluon density, soft gluon and soft + hard gluon, as in the dipole case. However, the results of the HERAPDF fit in the low $x$ region only are sizably scheme dependent. In the standard HERAPDF RT-OPT scheme [11] the fit quality is somewhat poorer, $\chi^{2} / N_{d f}=1.06$, instead of $\chi^{2} / N_{d f}=1.01$ in FONLL-B [12].

In Fig. 2 we show a comparison of the gluon density obtained in the fits with valence quarks and compare it to the gluon density obtained in the HERAPDF fit. We see that 
the two gluon densities differ, even substantially, at smaller scales but then start to closely approach each other at higher scales.

Summarizing we can tell that the dipole BGK fits describe the highest precision HERA data, in the low $x$ region and for $Q^{2}>3.5 \mathrm{GeV}^{2}$, very well. The best fits were obtained with the five parameter form of the gluon density and with the saturation ansatz, Table 4 and Figure 1. The differences in the fit quality, between various fits performed in this region are however pretty small. In our previous work [1], which used a subset of data from the present evaluation and had a much poorer evaluation of errors, the differences between the fits with and without valence quarks were more pronounced. The correlated errors of the final data are more restrictive than the uncorrelated one, as seen from the standard HERAPDF fits, however, in the case of dipole fits, the full treatment of errors makes the contribution of valence quarks less visible in the low $x$ region. It is also interesting to observe that in difference to the previous fits (with uncorrelated errors) [1], the present fits have a similar quality in the higher and lower $Q^{2}$ region. A substantial improvement of the fit quality with the increase of $Q^{2}$ cut, observed in the previous evaluation, like $\chi^{2} / N \approx 1$ for $Q^{2}>3.5 \mathrm{GeV}^{2}$ and $\chi^{2} / N \approx 0.8$ for $Q^{2}>8.5 \mathrm{GeV}^{2}$ [1], seemed to suggest some kind of saturation or lack of higher order QCD corrections. This is now not seen anymore, all fits seem to be of similar quality.

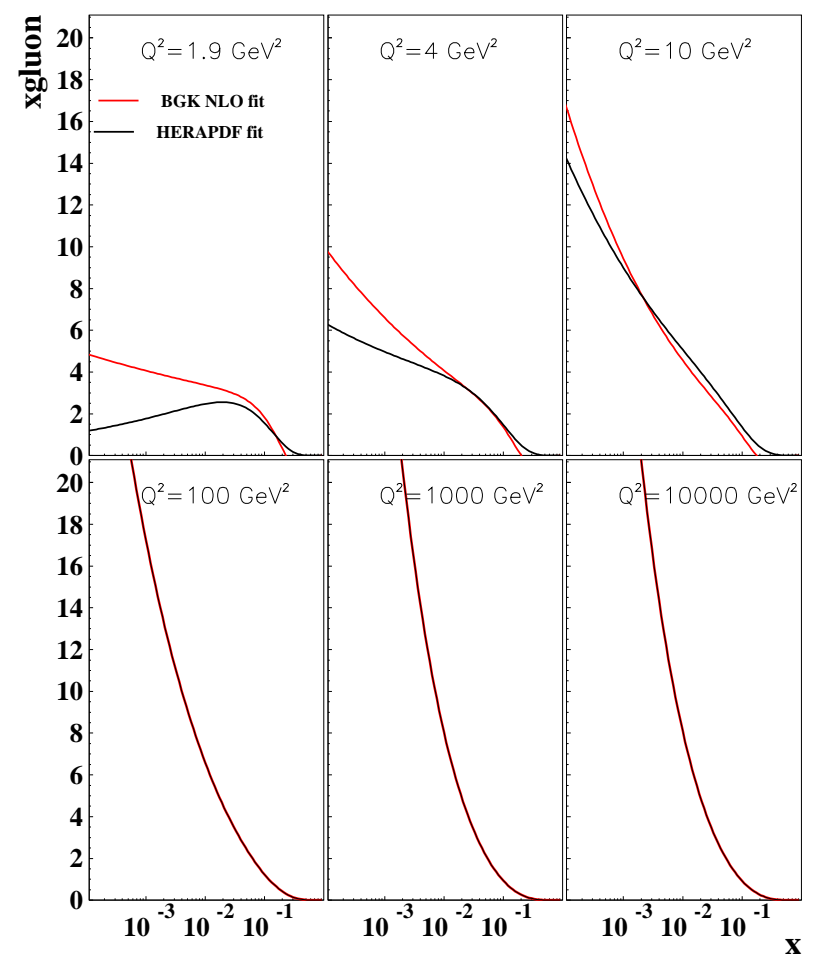

Figure 2: Comparison between the dipole (soft), Table 1, and HERAPDF gluon (soft) in NLO. 


\section{Investigation of saturation effects}

Saturation is a property of a gluonic state, which is so dense that gluons start to interact with each other. In the BGK dipole model, saturation effects are described in the eikonal approximation, eq. 2.3. To investigate the importance of saturation at HERA we performed also fits without the saturation ansatz, i.e when only the first term in the expansion of the exponent of eq. 2.3 is taken into account, i.e.

$$
\sigma_{\text {dip }}\left(x, r^{2}\right)=\pi^{2} r^{2} \alpha_{s}\left(\mu^{2}\right) x g\left(x, \mu^{2}\right) / 3 .
$$

In Table 10 we show the results of such a fit at two starting scales, $Q_{0}^{2}=1.9$ and $1.1 \mathrm{GeV}^{2}$. Both fits describe the data fairly well although the fit quality is slightly worse than in the fits with the saturation ansatz, Table 4 and 5 .

\begin{tabular}{|c|c|c|c|c|c|c|c|c|c|}
\hline$Q_{\min }^{2}\left[\mathrm{GeV}^{2}\right]$ & $Q_{0}^{2}\left[\mathrm{GeV}^{2}\right]$ & $A_{g}$ & $\lambda_{g}$ & $C_{g}$ & $D_{g}$ & $E_{g}$ & $N d f$ & $\chi^{2}$ & $\chi^{2} / N p$ \\
\hline 3.5 & 1.9 & $2.33 \pm$ & $-0.094 \pm$ & $14.8 \pm$ & $9.80 \pm$ & $-99.5 \pm$ & 533 & 556.17 & 1.04 \\
& & 0.10 & 0.006 & 11.5 & 14.7 & 74.830 & & & \\
\hline 3.5 & 1.1 & $3.80 \pm$ & $0.10 \pm$ & $32.5 \pm$ & $-25.2 \pm$ & $1868 \pm$ & 533 & 539.2 & 1.01 \\
& & 0.22 & 0.01 & 1.6 & 3.49 & 252 & & & \\
\hline
\end{tabular}

Table 10: BGK fit with valence quarks for $\sigma_{r}$ for H1ZEUS-NC data in the range $Q^{2} \geq$ $3.5 \mathrm{GeV}^{2}$ and $x \leq 0.01$. NLO fit. Soft + hard gluon. $m_{u d s}=0.14, m_{c}=1.3 \mathrm{GeV}$, non-saturation ansatz. $Q_{0}^{2}=1.9$ or $1.1 \mathrm{GeV}^{2}$.

We observe that the values of the parameter $\sigma_{0}$ of dipole cross section are quite high, of the order $70 \mathrm{mb}$ for $Q_{0}^{2}=1.9 \mathrm{GeV}^{2}$ and $220 \mathrm{mb}$ for $Q_{0}^{2}=1.1 \mathrm{GeV}^{2}$, see Table 4 and 5 . This is much higher than in the original GBW and BGK model fits [4,5], where this number was around $23 \mathrm{mb}$. This is an interesting result because $\sigma_{0}$ is the black disk limit of the dipole cross section, i.e. its value at very large energies. It indicates that the exponential form of the dipole cross section may be of little importance because, in the limit of very high values of $\sigma_{0}$, the dipole cross section reduces to the first term of the expansion of the exponent in the dipole cross section, see eq 4.1. This is in agreement with the fit results performed with and without saturation shown in Table 4, 5 and 10 .

In Figure 3 we compare the gluon densities obtained from fits with and without saturation ansatz of Tables 4 and 10. Note that for $x<0.001$, the gluon densities obtained in the dipole approach are higher than that of the standard pdf fit, Fig. 2, and that of the non-saturated one, see Fig 3 . This is expected and is due to damping of gluon density by saturation effects.

The fits of Table 4, 5 and 10 where performed in the higher $Q^{2}>3.5 \mathrm{GeV}^{2}$ region. Although they show some slight worsening of the fit quality between the saturated and nonsaturated case, the differences are by themselves too small to be considered as an indication of saturation. The situation changes however when we start to look in the region of smaller $Q^{2}$ 's, less than $3.5 \mathrm{GeV}^{2}$.

The degree of saturation, in DIS, is characterized by the size of the dipole, $r_{S}$, which, at a given $x$, starts to interact multiple times in a proton (in about $60 \%$ of cases). It is 


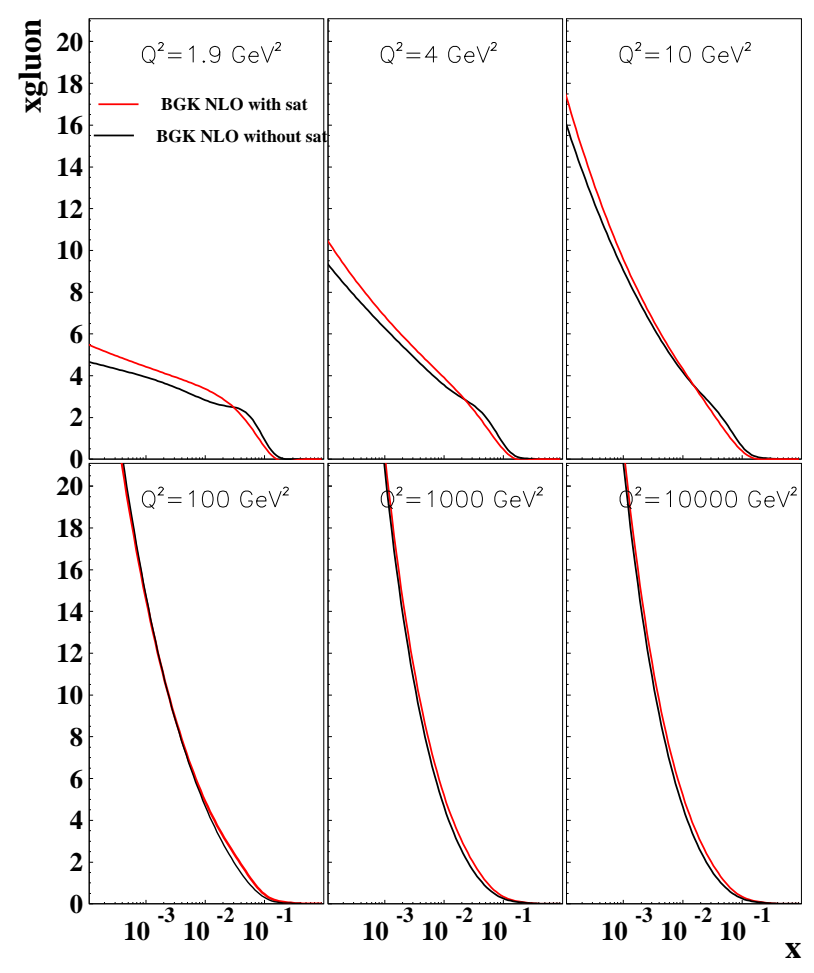

Figure 3: Comparison between the gluon densities obtained from fits in the $Q^{2}>3.5 \mathrm{GeV}^{2}$ region, with and without the saturation ansatz, Table 4 and 10 ,

usually expressed as a saturation scale $Q_{S}^{2}=2 / r_{S}^{2}$, which in the GBW model is given by $Q_{S}^{2}=2 / R_{0}^{2}$. In the BGK (or KMW) model it can be determined directly, from the gluon density, using eq. 4.17 (or its analog in KMW). In the complete, impact parameter dependent analysis of [9], it was determined at HERA as $Q_{S}^{2}=0.5 \mathrm{GeV}^{2}$ at $x=10^{-3}$ and as about 1 $\mathrm{GeV}^{2}$ at $x=10^{-4}$.

The low value of the saturation scale, $Q_{S}^{2}$, determined in [9], suggests that we should be looking for saturation effects in the low $Q^{2}$ data region. In Fig. 4, we show, therefore, the results of the extrapolation to a low $Q^{2}$ region, $Q^{2}<3.5 \mathrm{GeV}^{2}$, of the fits with and without saturation of Tables 4 and 10. We see that the fit with saturation of Table 4 (solid line) extrapolates down to $Q^{2}=0.85 \mathrm{GeV}^{2}$ fairly well. The extrapolation to even lower $Q^{2}$ starts to overshoot the data in a systematic way. The fit without saturation, of Table 10 (dashed line), extrapolates well to $Q^{2}=1.2 \mathrm{GeV}^{2}$ only. The extrapolation to the lower $Q^{2}$ region overshoots the data sizably stronger than in the fit with saturation. The $\chi^{2} / N_{d f}$ of the fit which uses the saturation ansatz (solid line), including the extrapolated points, is 1.24. For the fit without saturation (dashed line) it is 1.6.

We also fitted data with and without the saturation ansatz in the whole $Q^{2}$ region, $0.35<Q^{2}<250 \mathrm{GeV}^{2}$, and found that the fit is only slightly better than in the extrapolated case. The results are given in Table 11 for the saturated case and in Table 12 for the nonsaturated fit. For both starting scales, the fits with the saturated ansatz are sizably closer 


\begin{tabular}{|c|c|c|c|c|c|c|c|c|c|}
\hline$Q_{0}^{2}\left[\mathrm{GeV}^{2}\right]$ & $\sigma_{0}[\mathrm{mb}]$ & $A_{g}$ & $\lambda_{g}$ & $C_{g}$ & $D_{g}$ & $E_{g}$ & $N_{d f}$ & $\chi^{2}$ & $\chi^{2} / N_{d f}$ \\
\hline 1.9 & $38.2 \pm$ & $2.80 \pm$ & $-0.063 \pm$ & $46.3 \pm$ & $12.1 \pm$ & $1970.4 \pm$ & 653 & 790.4 & 1.21 \\
& 4.1 & 0.14 & 0.006 & 4.58 & 6.00 & 566.0 & & & \\
\hline 1.1 & $196,1 \pm$ & $6.24 \pm$ & $0.098 \pm$ & $52.3 \pm$ & $-22.0 \pm$ & $2145.0 \pm$ & 653 & 894.1 & 1.37 \\
& 105 & 0.53 & 0.012 & 6.5 & 10.64 & 835.7 & & & \\
\hline
\end{tabular}

Table 11: BGK fit with valence quarks for $\sigma_{r}$ for H1ZEUS-NC data in the range $Q^{2} \geq$ $0.35 \mathrm{GeV}^{2}$ and $x \leq 0.01$. NLO fit. Soft + hard gluon. $m_{u d s}=0.14, m_{c}=1.3 \mathrm{GeV}$, saturation ansatz. $Q_{0}^{2}=1.9$ or $1.1 \mathrm{GeV}^{2}$.

\begin{tabular}{|c|c|c|c|c|c|c|c|c|}
\hline$Q_{0}^{2}\left[\mathrm{GeV}^{2}\right]$ & $A_{g}$ & $\lambda_{g}$ & $C_{g}$ & $D_{g}$ & $E_{g}$ & $N_{d f}$ & $\chi^{2}$ & $\chi^{2} / N_{d f}$ \\
\hline 1.9 & $3.05 \pm$ & $-0.022 \pm$ & $40.3 \pm$ & $-32.3 \pm$ & $3158.3 \pm$ & 654 & 1024.3 & 1.56 \\
& 0.092 & 0.004 & 1.067 & 3.02 & 219.3 & & & \\
\hline 1.1 & $5.62 \pm$ & $0.158 \pm$ & $43.320 \pm$ & $-55.011 \pm$ & $3791.6 \pm$ & 654 & 999.98 & 1.53 \\
& 0.13 & 0.001 & 0.15 & 8.62 & 187.7 & & & \\
\hline
\end{tabular}

Table 12: BGK fit with valence quarks for $\sigma_{r}$ for H1ZEUS-NC data in the range $Q^{2} \geq$ $0.35 \mathrm{GeV}^{2}$ and $x \leq 0.01$. NLO fit. Soft + hard gluon. $m_{u d s}=0.14, m_{c}=1.3 \mathrm{GeV}$, non-saturation ansatz. $Q_{0}^{2}=1.9$ or $1.1 \mathrm{GeV}^{2}$.

to data, which indicates a presence of saturation effects. The best fit is obtained with the saturated fit of Table 11, at the QCD scale $Q_{0}^{2}=1.9$, which has $\chi^{2} / N_{d f}=1.21$. The value of the parameter $\sigma_{0}$, which is a black disc limit of virtual photon-proton cross section, has the smallest error and is within 2 standard deviations consistent with the fit at higher $Q^{2}$, of Table 4. Its value of around $40 \mathrm{mb}$ is also close to the value of the same parameter obtained in previous fits [4,5], which were around $20 \mathrm{mb}$.

In the saturation investigation of ref [5, 8], were the first set of HERA data was used, the fits with and without saturation had the same quality, even when the low $Q^{2}$ region was included in the fit. Therefore, the present result that the fit with saturation has a sizably better quality than that without saturation is new and is due to the substantially improved quality of HERA data.

Finally let us also note, that the lack of very good description of data, expressed by the worsening of the $\chi^{2} / N_{d f}$ value from 1.00 to 1.21 when the lower $Q^{2}$ region is included, together with the systematic overshoot of the fits over data observed in Fig. 4 at $Q^{2}<1 \mathrm{GeV}^{2}$, suggests that the approach to saturation realized in the BGK model may be too crude. The large discrepancies between the values of $\sigma_{0}$ parameter of Tables 11, 4 and 5 together with their large measurement errors suggest that the saturation investigation presented here should be extended using an impact parameter dependent dipole model [8, 9]. In such a model, the QCD evolution and the saturation ansatz is the same as in the BGK model, however, the $\sigma_{0}$ parameter is replaced by the transverse profile of the proton. The proton profile, which determines the impact parameter distribution, is obtained from the data of the exclusive diffractive scattering of vector mesons. Therefore, there is effectively one free 
parameter less, which could lead to an improved investigation of the saturation mechanism. We intend to come back to this subject in an extended evaluation of HERA data which will also include the final, exclusive diffractive scattering HERA data. The value of the investigation performed in this paper lays in its simplicity; in spite of the fact that the BGK model has one free parameter more than the impact parameter dependent model, it provides a clear evidence that saturated dipole mechanism is closer to data than the non-saturated one.

\section{Summary}

We found that the dipole BGK fits with the DGLAP QCD evolution describe the highest precision HERA data, in the low $x$ region and for $3.5<Q^{2}>250 \mathrm{GeV}^{2}$, very well. The best fits were obtained with the five parameter form of the gluon density and with the saturation ansatz, Table 4 and Figure 1. The differences in the fit quality, between various fits performed in this region are however pretty small.

The present paper is focused on the saturation question, which is investigated by various fits to data with and without the saturation ansatz. The fits were made with different gluon densities and using different QCD starting scales. All dipole fits to data in the higher $Q^{2}>3.5 \mathrm{GeV}^{2}$ region, are describing data very well. No significant differences between the saturated and no-saturated fits were observed. The fits to data including the lower $0.35<Q^{2}<3.5 \mathrm{GeV}^{2}$ region, show however, that the saturated gluon density is preferred. This is a new result, which is due to a substantial improvement of data quality obtained in the final evaluation of HERA data. It indicates also that there is more information about saturation in HERA data, which should be evaluated using impact parameter dependent dipole models.

\section{Acknowledgement}

We would like to thank Sasha Glazov (DESY) for various help and useful discussions from the beginnig of the analysis presented here till the final version of the manuscript. We also thank xFitter developers' group for useful comments and Pavel Belov for careful reading of the manuscript. This work is supported by the Polish Ministry under program Mobility Plus, no. 1320/MOB/IV/2015/0.

\section{References}

[1] H. Kowalski, A. Luszczak, Phys. Rev. D 89, 074051 (2014)

[2] F. D. Aaron et al. [H1 and ZEUS Collaborations], JHEP 1001 (2010) 109.

[3] H. Abramowicz et al. [H1 and ZEUS Collaborations], Eur. Phys. J. C75 (2015) nb.12. 


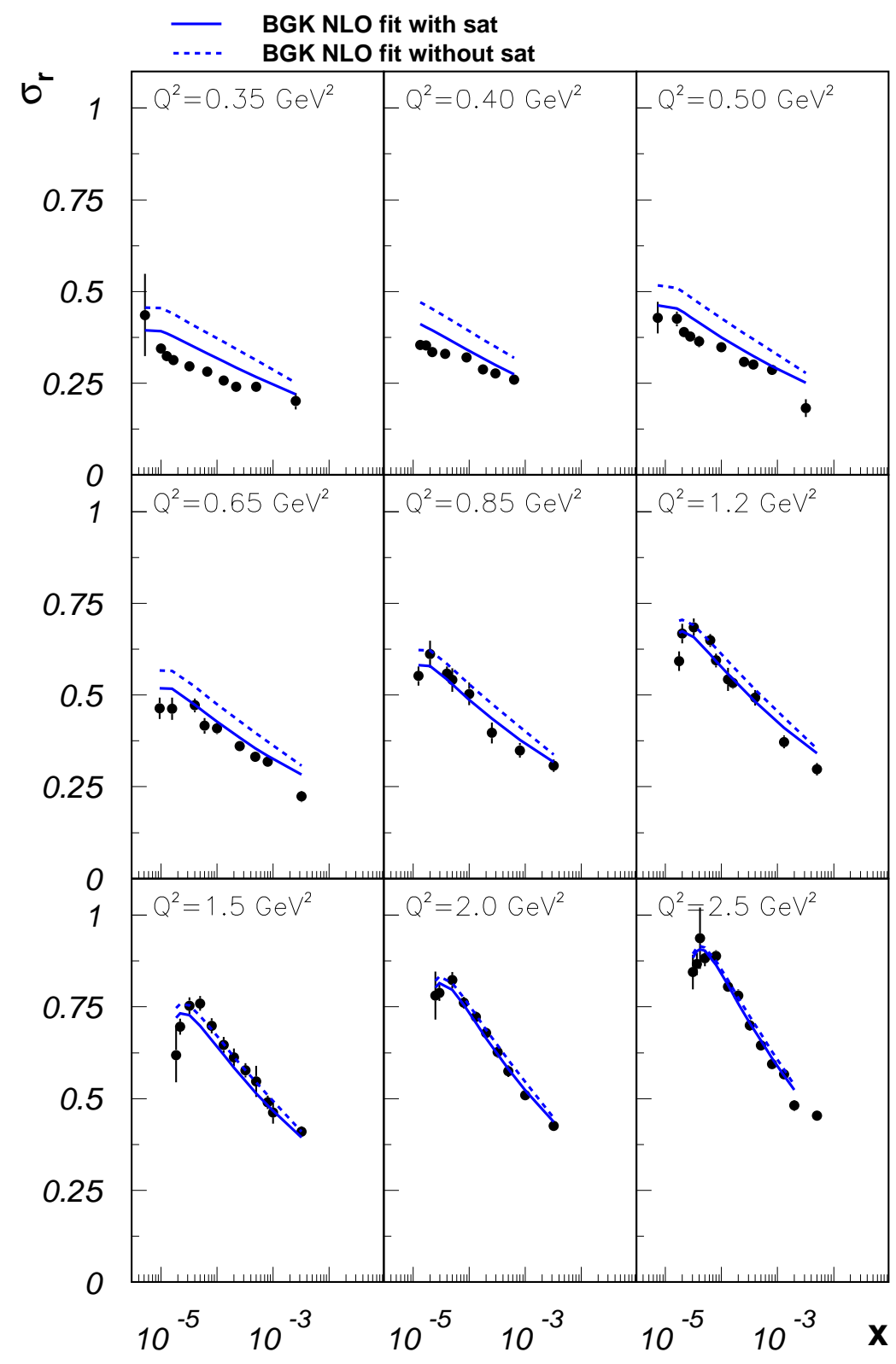

Figure 4: Comparison of the extrapolated dipole BGK fit of Table 4 and 10 with the reduced cross sections of the final, combined H1 and ZEUS HERA data. For better visibility only $920 \mathrm{GeV}$ data are displayed. The starting scale of the evolution was $Q_{0}^{2}=1.9 \mathrm{GeV}^{2}$. The fit was performed in the $Q^{2}>3.5 \mathrm{GeV}^{2}$ region and then extrapolated to the lower $Q^{2}$ region seen in the figure. Solid line shows the extrapolation of the fit with saturation and the dashed one without saturation. 
[4] K. Golec-Biernat, M. Wuesthoff, Phys. Rev. D59, 014017 (1999); Phys. Rev. D60, 114023 (1999).

[5] J. Bartels, K.Golec-Biernat and H. Kowalski, Phys.Rev. D66, (2002) 19

[6] E. Iancu, K. Itakura, and S. Munier. Phys. Lett., B590:199-208, 2004.

[7] S. Munier, A. M. Staśto and A. H. Mueller, Nucl. Phys. B 603 (2001) 427.

[8] H. Kowalski and D. Teaney, Phys. Rev. D68 (2003) 114005.

[9] H. Kowalski, L. Motyka and G. Watt, Phys. Rev. D74 (2006) 074016.

[10] "HERAFitter, Open Source QCD Fit Project" By S. Alekhin at al., DESY Report 14-188, 7 Nov 2014, Published in EPJC (2015), 75: 304, e-Print: arXiv:1410.4412 [hep-ex]

xfitter.org

"Combination of measurements of inclusive deep inelastic ep scattering cross sections and QCD analysis of HERA data." By H1 and ZEUS Collaborations (H. Abramowicz et al.). DESY-15-039, Jun 19, 2015. 160 pp. Published in EPJC (2015) 75: 12. e-Print: arXiv:1506.06042 [hep-ex]

"Fast QCD Evolution and Convolution", M. Botje, NIKHEF-10-002, May 2010. 74pp. Published in Comput.Phys.Commun.182:490-532,2011. e-Print: arXiv:1005.1481 [hep-ph]

F. James, M. Roos (CERN). Jul 1975. 38 pp. Published in Comput.Phys.Commun. 10 (1975) 343-367 CERN-DD-75-20 DOI: 10.1016/0010-4655(75)90039-9

"An Ordered analysis of heavy flavor production in deep inelastic scattering" R.S. Thorne, R.G. Roberts. RAL-TR-97-049, Sep 1997. 48pp. Published in Phys.Rev.D57:6871-6898,1998. e-Print: hep-ph/9709442

"A Variable-flavor number scheme for NNLO" R.S. Thorne. CAVENDISH-HEP-200601, Jan 2006. 17pp. Published in Phys.Rev.D73:054019,2006. e-Print: hep-ph/0601245

"MeasurementoftheInclusiveepScatteringCross SectionatLow $Q^{2}$ andxat HERA"

F.D. Aaron et al. DESY-08-171, 2009. 90pp. Published in Eur.Phys.J.C63:625678,2009. e-Print: arXiv:0904.0929 [hep-ex]

"An NLO QCD analysis of inclusive cross-section and jet-production data from the zeus experiment" By ZEUS Collaboration (S. Chekanov et al.). DESY-05-050, Mar 2005. 37pp. Published in Eur.Phys.J.C42:1-16,2005. e-Print: hep-ph/0503274

"Multivariate fitting and the error matrix in global analysis of data" J. Pumplin, D.R. Stump, W.K. Tung. MSU-HEP-07100, CERN-TH-2000-249, Aug 2000. 14pp. 
Published in Phys.Rev.D65:014011,2001. e-Print: hep-ph/0008191

"New generation of parton distributions with uncertainties from global QCD analysis" J. Pumplin, D.R. Stump, J. Huston, H.L. Lai, Pavel M. Nadolsky, W.K. Tung, MSU-HEP-011101, Jan 2002. 44pp. Published in JHEP 0207:012,2002. e-Print: hep-ph/0201195

[11] "The Effect of Changes of Variable Flavour Number Scheme on PDFs and Predicted Cross Sections" R.S. Thorne. Jan 2012. 14pp. Published in Phys.Rev. D86 (2012) 074017 e-Print: arXiv:1201.6180 [hep-ph]

[12] "APFEL: A PDF Evolution Library with QED corrections" V. Bertone, S. Carrazza and J. Rojo, Published in Comput. Phys. Commun. 185 (2014) 1647, e-Print: arXiv:1310.1394 [hep-ph].

"Heavy quarks in deep-inelastic scattering" S. Forte, E. Laenen, P. Nason and J. Rojo, Published in Nucl. Phys. B 834 (2010) 116, e-Print: arXiv:1001.2312 [hep-ph].

[13] N. N. Nikolaev and B. G. Zakharov, Z. Phys. C49 (1991) 607; Z. Phys. C53 (1992) 331.

[14] J. Nemchik, N. N. Nikolaev, E. Predazzi and B. G. Zakharov, Z. Phys. C 75 (1997) 71.

[15] E. Gotsman, E. Levin and U. Maor, Nucl. Phys. B 464 (1996) 251.

[16] H. G. Dosch, T. Gousset, G. Kulzinger and H. J. Pirner, Phys. Rev. D 55 (1997) 2602.

[17] A. C. Caldwell and M. S. Soares, Nucl. Phys. A 696, 125 (2001).

[18] J. R. Forshaw, R. Sandapen and G. Shaw, Phys. Rev. D 69 (2004) 094013.

[19] L. Frankfurt, M. Strikman and C. Weiss, Ann. Rev. Nucl. Part. Sci. 55 (2005) 403.

[20] H. Kowalski, T. Lappi, C. Marquet and R. Venugopalan, Phys. Rev. C78 (2008) 045201. 